\title{
7(6)-羟基黄酮衍生物的合成及初步抗肿瘤活性研究
}

\author{
王学军*, $a$ 刘建利 $b$ \\ $\left({ }^{a}\right.$ 陕西省商洛学院生物医药工程系 商洛 726000) \\ $\left({ }^{b}\right.$ 西部资源生物与现代生物技术省部共建教育部重点实验室 西安 710069)
}

\begin{abstract}
摘要 以间苯二酚和对苯二酚为起始原料, 经过傅克酰化、Baker-Venkataraman 重排、关环三步反应分别得到 5 个 7 羟基黄酮和 5 个 6-差基黄酮类化合物. 试验结果发现，这些化合物溶解性能不好，生物活性不高，因此，对这些化合物 又经过苯甲酰化并将 7-羟基黄酮与 1-氯-3-溴丙烷首先发生亲核取代反应，然后和诺氟沙星在丙酮中反应生成化合物 13, 总共得到了 5 个结构新颖的黄酮和 4 个未见文献报道的 $\beta$-丙二酮类化合物. 通过四甲基偶氮唑盐(MTT)法对 19 个 化合物的抑制人宫颈癌细胞 Hela 活性进行了体外评价, 结果表明: 化合物 $\mathbf{1 3}$ 的半数抑制浓度 $\mathrm{IC}_{50}$ 为 $19.4 \mu \mathrm{mol} / \mathrm{L}$, 具 有深入研究的价值.
\end{abstract}

关键词 7(6)-羟基黄酮; 衍生物; 合成; 抗肿瘤活性

\section{Synthesis and Preliminary Antitumor Activities of 7(6)-Hydroxylflavone Derivatives}

\author{
Wang, Xuejun*,a Liu, Jianli ${ }^{b}$ \\ $\left({ }^{a}\right.$ Department of Biomedical Engineering, Shangluo University, Shangluo 726000) \\ $\left({ }^{b}\right.$ Key Laboratory of Resource Biology and Biotechnology in Western China, Ministry of Education, \\ School of Life Science, Northwest University, Xi'an 710069)
}

\begin{abstract}
Five 7-hydroxylflavones and five 6-hydroxylflavones have been synthesized with resorcinol and paradioxybenzene as starting materials in three steps by an improved Baker-Venkatarama rearrangement reaction. Experimental results showed that the ten compounds have poor solubility in methanol and chloroform solvent and low biological activity. Therefore, some of these compounds are further derivatived and five new compounds were successfully synthesized. The nineteen compounds, including four new $\beta$-propenediones $\mathbf{4 b}, \mathbf{4 c}, \mathbf{4 d}$ and $\mathbf{4 e}$, were evaluated for antitumor activities against hela human cervical carcinoma cell lines by methyl thiazolyl tetrazolium (MTT) method. The preliminary biological activity tests showed that compound $\mathbf{1 3}$ exhibited potentially high activity against hela human cervical carcinoma cell lines with $\mathrm{IC}_{50}$ value of 19.4 $\mu \mathrm{mol} / \mathrm{L}$.
\end{abstract}

Keywords 7(6)-hydroxyflavone; derivative; synthesis; antitumor activity

黄酮类化合物广泛分布于自然界，为植物的一类次 生代谢产物, 具有广谱的生理活性 ${ }^{[1 \sim 3]}$. 但是, 由于黄 酮类化合物的溶解性能差, 生物利用度低, 再加上生理 活性极其广泛，作用靶点多，缺乏对病症治疗的选择性， 诸如此类现象是限制黄酮类化合物直接开发成新药的 瓶颈问题. 因此, 利用化学合成的方法对黄酮类化合物 进一步结构修饰和衍生化, 以解决上述问题给黄酩类新
药开发带来的困难具有很大的现实意义. 此外，黄酮类 化合物还是许多中草药黄芩、银杏、沙棘等的主要活性 成分, 对于此类化合物的合成和药效研究, 有助于我们 正确理解中药治病、防病的作用机制，指导我们正确用 药.

黄酮类化合物的合成方法主要有两种，即查尔酮路 线和 $\beta$-丙二酮路线 (Baker-Venkataraman 法 $)^{[4,5]}$. 经典的

\footnotetext{
*E-mail: xuejunwangd@163.com

Received February 11, 2014; revised March 25, 2014; published online April 18, 2014

Project supported by the National Natural Science Fundation of China (Nos. 20872118, 30070905), the Natural Science Fundation of Shaanxi Province (No. 2014JM4097), the Foundation of the Education Department of Shaanxi Province (No. 2013JK0836) and the Talent Introduction Foundation of Shangluo University (No. 13sky0035).

国家自然科学基金(Nos. 20872118, 30070905)、陕西省自然科学基金(No. 2014JM4097)、陕西省教育厅基金(No. 2013JK0836)、商洛学院人才引进基 金(No. 13sky0035)资助项目.
} 
Baker-Venkataraman 重排反应分三步进行, 分别是酯 化、重排和关环, 最终得到黄酮类成分 ${ }^{[6,7]}$. 所谓重排是 指 $\alpha$-苯甲酰氧基苯乙酮在碱性条件下发生分子内 Claisen 缩合重排生成 1,3-丙二酮类化合物, 是 BakerVenkataraman 反应中最为关键的一步. Chee 等 ${ }^{[8]}$ 发现, 将 2-差基苯乙酮及其衍生物和苯甲酰氯在碳酸钾的丙 酮溶液中加热回流 $24 \mathrm{~h}$ 后, 结果发现, Baker-Venkataraman 反应中的酯化、重排和关环三步反应一步完成, 直接生成了两种黄酮类化合物.

在黄酮类化合物的众多生物活性之中, 抗肿瘤的活 性最为引人注目 ${ }^{[9]}$, 其抗菌活性已经得到了医学界的广 泛认可, 文献报道了许多具有较好抗菌活性的天然黄酮 类化合物 ${ }^{[10]}$. 但我们的试验结果显示, 黄酮类化合物单 用时, 抗菌作用并不明显, 最近有文献报道, 黄酤类化 合物和抗生素具有协同抗菌作用 ${ }^{[11 ~ 13]}$, 研究最多的是 黄烷-3-醇, 例如, 表儿茶素没食子酸酯能够减小 $\beta$-内酰 胺类抗生素对耐甲氧西林金黄色葡萄球菌(MRSA)的最 小抑菌浓度(MICs) 512 倍多 ${ }^{[14]}$. 农朝赞等 ${ }^{[15]}$ 发现芒果苷 单用时对痤疮丙酸杆菌和金黄色葡萄球菌的抑菌效果 不明显, MIC 值分别为 3378.4 和 $8446.0 \mathrm{mg} / \mathrm{L}$. 但与红霉 素联合使用时, 能够降低红霉素的使用量, $0.117 \mathrm{mg} / \mathrm{L}$ 的红霉素和 $42.23 \mathrm{mg} / \mathrm{L}$ 的芒果苷配伍使用, 就对上述两 种致病菌显示抑制作用.

在上述文献的启发下, 我们设计利用化学方法将将 抗生素和黄酮体进行偶联, 期望偶联后的成分能产生协 同抗菌的作用, 但实验结果显示, 7-羟基黄酩和抗生素 诺氟沙星偶联后的化合物 13, 其抗菌活性不但没有增 强反而降低了诺氟沙星的抗菌作用, 然而意外发现, 化 合物 13 具有潜在的抗肿瘤活性, 对 $\mathrm{HeLa}$ 细胞的 $\mathrm{IC}_{50}$ 值 为 $19.4 \mu \mathrm{mol} / \mathrm{L}$, 具有深入研究的价值.

本项目对 Baker-Venkataraman 反应进行系统研究的 基础上, 对其又进行了改进, 改进后的合成方法可以一 锅法合成黄酩体，为该类化合物生物活性的研究奠定了 基础.

\section{1 结果与讨论}

\section{1 化合物的合成}

以不同的原料对改进的 Baker-Venkataraman 重排反 应进行了系统研究，结果发现，当 2-羟基苯乙酮的 6-位 没有羟基时，例如 2-差基苯乙酮、2,4-二羟基苯乙酮、 2,5-二羟基苯乙酮、3-甲基-2,5-二羟基苯乙酮等和苯甲 酰氯及其衍生物在碳酸钾的丙酮溶液中发生 Baker-Venkataraan 重排反应，其酯化、重排一步完成 首先得到 $\beta$-丙二酮类化合物, 将 $\beta$-丙二酮关环就可得 到黄酮类化合物; 当 2-看基苯乙酮的 6 -位有着基时,
例如 2,6-二羟基苯乙酮及 2,4,6-三羟基苯乙酮，它们 与苯甲酰氯及其衍生物在碳酸钾的丙酮溶液中也可 发生 Baker-Venkataraman 重排反应，并且酯化、重排、 关环一步完成，直接得到 5-羊基黄酮、3-苯甲酰基-5羟基黄酮及其衍生物和 3-苯甲酰基-5,7-二差基黄酮 等.

本文对碳酸钾催化下的 Baker-Venkataraman 重排反 应又进行了改进，实验发现，在无水碳酸钾的催化下， 以丙酮作为溶剂, 再加入少量吡啶后, 不管 2 -差基苯乙 酮的 6-位是否有羟基，例如 2,4-二羟基苯乙酮、2,5-二羟 基苯乙酮、3-甲基-2,5-二羟基苯乙酮、2,6-二羟基苯乙 酮及 2,4,6-三差基苯乙酮等和苯甲酰氯及其衍生物反应, 其酯化、重排、关环一步完成，一锅法一步合成得到相 应的黄酮类化合物, 该方法正在申请发明专利之中.

在实验过程中对 $\beta$-丙二酮类化合物关环反应中常 用的几种关环试剂进行了篮选和优化, 发现用 HOAc$\mathrm{H}_{2} \mathrm{SO}_{4}$ 体系关环且当 $\mathrm{H}_{2} \mathrm{SO}_{4}$ 和 $\mathrm{HOAc}$ 体积比为 $0.8: 100$ 时黄酮类化合物的收率最高.

6-羊基黄酮及其衍生物的合成反应原理如图 1 所 示: 以对苯二酚为起始原料, 氯化锌为催化剂, 醋酸为 酰化剂通过傅克酰基化反应生成 2,5-二羟基苯乙酮. 在 碳酸钾的丙酮溶液中通过改进的 Baker-Venklataraman 重排得到 $\beta$-丙二酮，酸化环合生成了 5 个 6 -羊基黄酮及 其衍生物. 为了增加 6-差基黄酮及其衍生物的脂溶性, 将其和苯甲酰氯在碳酸钾的丙酮溶液中反应, 得到了两 个未见文献报道的 6-苯甲酰氧基黄酮类化合物 $6 \mathrm{~b}$ 和 $6 \mathrm{~d}$.

7-羊基黄酮及其衍生物的合成反应原理如图 2 所 示: 以间苯二酚为起始原料, 氯化锌为催化剂, 醋酸为 酰化剂通过傅克酰基化反应生成 2,4-二羟基苯乙酮. 然 后在丙酮中通过改进的 Baker-Venkataraman 重排反应 得到 5 个 7-羊基黄酮及其衍生物. 为增加 7-羟基黄酮及 其衍生物的脂溶性, 将其和苯甲酰氯在碳酸钾的丙酮溶 液中反应, 得到了两个未见文献报道的 7-苯甲酰氧基黄 酮类化合物 $11 \mathrm{~b}$ 和 $11 \mathrm{e}$; 为了增加 7-羊基黄酮及其衍生 物的水溶性和药效作用, 设计将具有较好水溶性、强极 性和强抗菌活性的第三代喹诺酮类抗菌剂诺氟沙星连 在 7-羊基黄酮上. 将 7-羊基黄酮在丙酮中和 1-氯-3-溴丙 烷发生亲核取代反应生成 $\mathbf{1 2}$. 再将 $\mathbf{1 2}$ 在丙酮中和诺氟 沙星反应最终得到化合物 13. 整个合成路线 7 步，高分 辨质谱和核磁共振氢谱数据表明诺氟沙星被连在了 7差弪基黄酮上.

\section{2 生物活性}

对于目标化合物 $5 \mathrm{a} \sim 5 \mathrm{e}, 10 \mathrm{a} \sim 10 \mathrm{e}, 6 \mathrm{~b}, 6 \mathrm{~d}, 11 \mathrm{~b}$, $11 \mathrm{e}, 13$ 及新化合物 $\beta$-丙二酮 $4 \mathrm{~b} \sim 4 \mathrm{e}$, 采用四甲基偶氮 唑盐(MTT)微量酶反应比色法测定了黄酮衍生物对人宫 


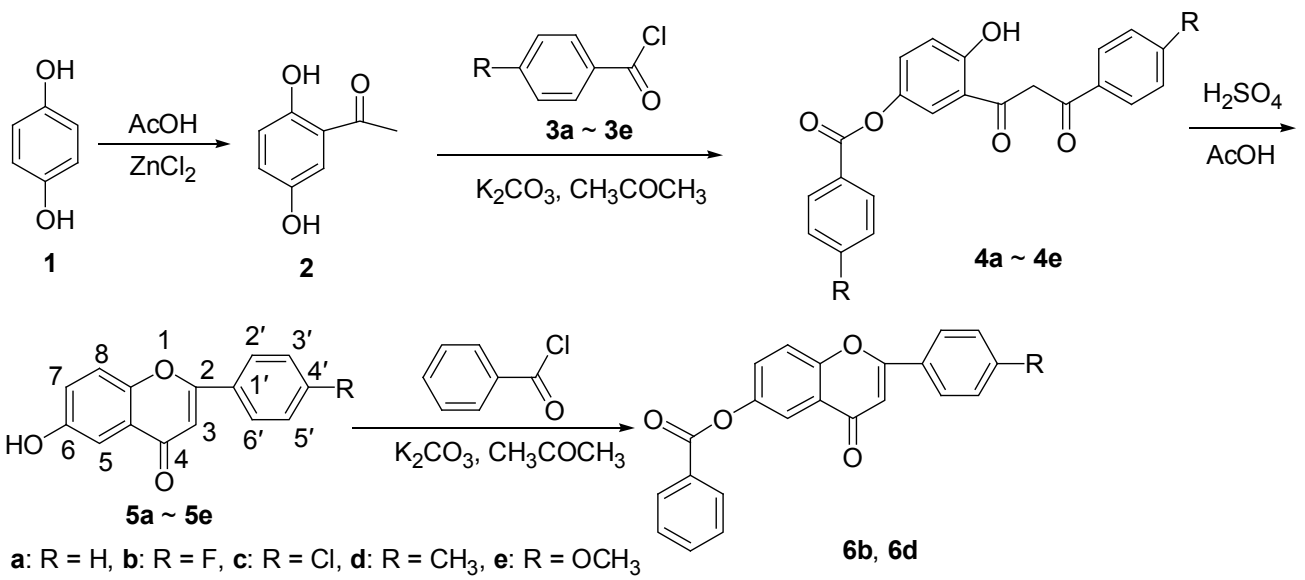

图 1 6-羊基黄酮衍生物的合成路线

Figure 1 Synthesis of 6-hydroxylflavones derivatives

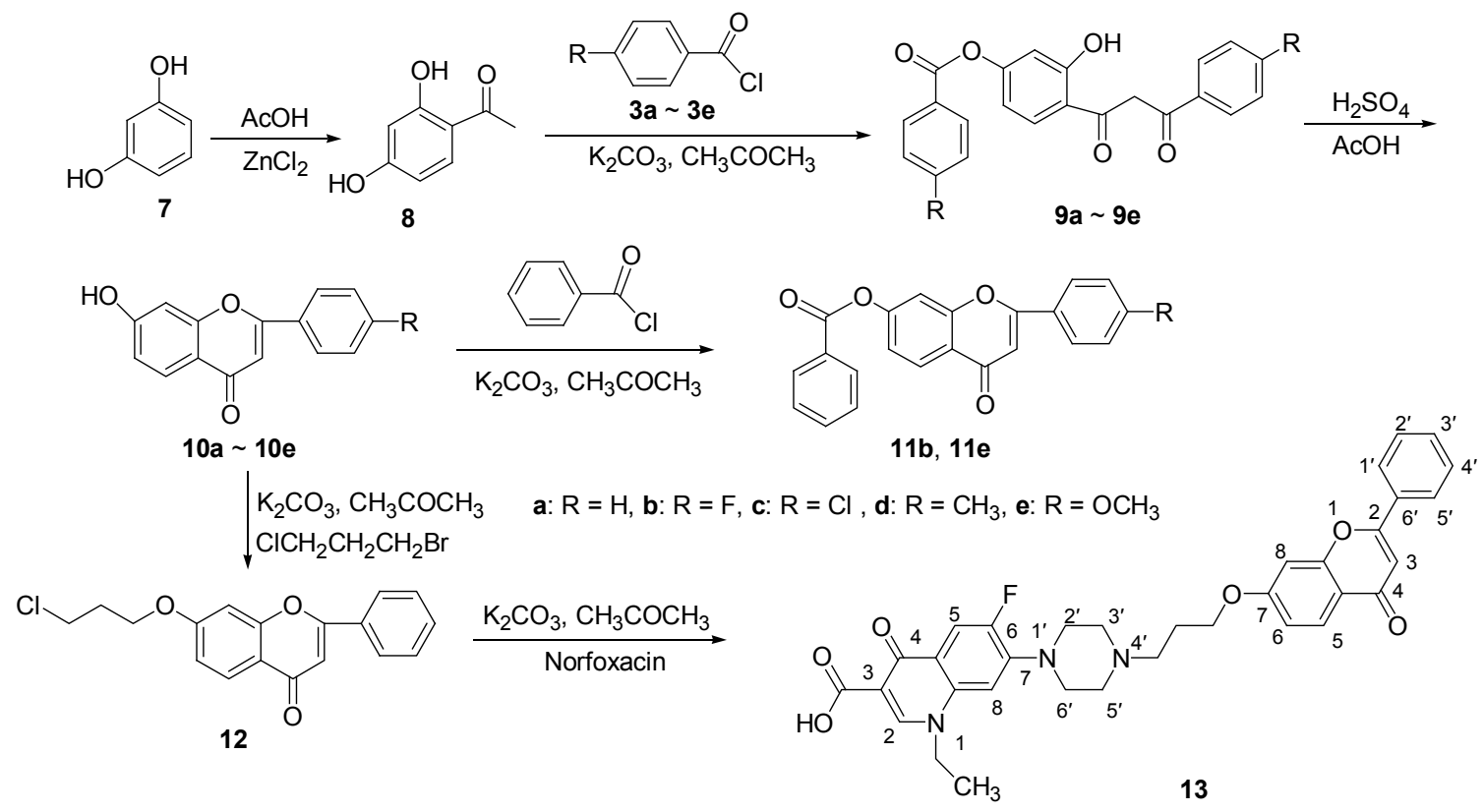

图 2 7-差基黄酮衍生物的合成路线

Figure 2 Synthesis of 7-hydroxylflavones derivatives

颈癌细胞(Hela)的体外抗肿瘤活性, 测试结果如表 1 所 示. 7-差基黄酮、6-羊基黄酮以及在它们母核的 4'-位分 别引入 $\mathrm{F}, \mathrm{Cl}, \mathrm{OCH}_{3}, \mathrm{CH}_{3}$ 后都无明显的抗肿瘤活性, 在 实验过程中发现, 这些黄酮体它们的溶解性能都很差, 为了增强其脂溶性, 我们设计将 7-羊基黄酮和 6-羊基黄 酮的着基苯甲酰化, 实验结果发现, 苯甲酰化后的化合 物 6b, 6d, 11b, 11e 虽然脂溶性有所改善, 但从表 1 可以 看出, 其抗肿瘤的活性并未增强.

为了增强 7-羊基黄酤及其衍生物的水溶性和药效 作用, 设计将具有较好水溶性、强极性和强抗菌活性的 第三代喹诺酮类抗菌剂诺氟沙星连在 7-美基黄酮上. 诺 氟沙星具有很强的极性和较好的水溶性, 薄层色谱显 示, 即使用甲醇作展开剂, 诺氟沙星几乎还是保持在原
点不动. 因此期望将它连在黄酩上以增强黄酮类成分的 水溶性和在胃肠道的吸收，从而提高生物利用度. 诺氟 沙星为第三代喹诺酮类抗菌剂, 具有抗菌谱广、抗菌力 强的特点. 期望它和黄酮相连后, 可产生协同增效作用, 从而增强黄酮类化合物抗肿瘤、抗菌的药效. 实验结果 发现, 化合物 13 的抗菌作用与诺氟沙星相比反而有所 下降, 但对 Hela 细胞具有较强的抗增殖活性, 其 $\mathrm{IC}_{50}$ 值 为 $19.4 \mu \mathrm{mol} / \mathrm{L}$, 该化合物详细的构效关系研究还在进 行之中.

\section{2 结论}

近年来, 我们课题组一直致力于黄酮类化合物的结 构改造和生物活性研究，实验结果显示，黄酮类化合物 
表 1 部分目标化合物的体外抗肿瘤活性

Table 1 In vitro antitumor activities of some target compounds against Hela cell line

\begin{tabular}{cc||cc}
\hline Compd. & $\mathrm{IC}_{50} /\left(\mu \mathrm{mol} \cdot \mathrm{L}^{-1}\right)$ & Compd. & $\mathrm{IC}_{50} /\left(\mu \mathrm{mol} \cdot \mathrm{L}^{-1}\right)$ \\
\hline $\mathbf{4 b}$ & $>100$ & $\mathbf{6 d}$ & $>100$ \\
$\mathbf{4 c}$ & $>100$ & $\mathbf{1 0 a}$ & $>100$ \\
$\mathbf{4 d}$ & $>100$ & $\mathbf{1 0 b}$ & $>100$ \\
$\mathbf{4 e}$ & $>100$ & $\mathbf{1 0 c}$ & $>100$ \\
$\mathbf{5 a}$ & $>100$ & $\mathbf{1 0 d}$ & $>100$ \\
$\mathbf{5 b}$ & $>100$ & $\mathbf{1 0 e}$ & $>100$ \\
$\mathbf{5 c}$ & $>100$ & $\mathbf{1 1 b}$ & $>100$ \\
$\mathbf{5 d}$ & $>100$ & $\mathbf{1 1 e}$ & $>100$ \\
$\mathbf{5 e}$ & $>100$ & $\mathbf{1 3}$ & 19.4 \\
$\mathbf{6 b}$ & $>100$ & & \\
\hline
\end{tabular}

的溶解性能不好, 生物活性不高, 所以在本工作中我们 利用改进的 Baker-Venkataraman 反应合成了系列 7(6)差基黄酮. 为了增强黄酮体的脂溶性，我们将黄酮体的 着基苯甲酰化. 实验结果表明, 黄酮体的脂溶性有所改 善，在氯仿中易溶，但其抗肿瘤和抗菌活性并未得到增 强; 同时，为了增强 7(6)-羟基黄酮的极性和水溶性，进 而达到增强其生物活性的目的, 我们将黄酮体和诺氟沙 星进行了偶联, 偶联后的化合物 13 水溶性较黄酮体有 所增强，微溶于甲醇，生物活性实验结果显示化合物 $\mathbf{1 3}$ 抗菌活性跟诺氟沙星相比有所下降, 但其表现出了较强 的抗肿瘤活性, 值得进一步深入研究.

\section{3 实验部分}

\section{1 仪器与试剂}

X-5 显微熔点测定仪(温度计未校正, 北京泰克仪器 有限公司); LTQ-XL 电喷雾电离质谱(美国 Thermo Finnigan 公司); Bruker Avance-400 MHz 核磁共振仪(TMS 作内标, $\mathrm{CDCl}_{3}$ 为溶剂, 瑞士 Bruker 公司); APEX-2 高分 辨质谱仪(德国布鲁克公司); EL311 型酶标免疫检测仪 (美国 Bio Tek 公司); 3164 型水套式 $\mathrm{CO}_{2}$ 稃箱(美国 Forma Scientific 公司); IX71 型倒置苂光相差显微镜(日 本 Olympus 公司). 四甲基偶氮唑盐(MTT)购于瑞士 Fluka 公司; RPMI-1640 培养基购于美国 HyClone 公司; 试验所用试剂均购于阿拉丁试剂公司, 均为分析纯.

实验所用人宫颈癌细胞株 Hela 由第四军医大学 惠赠.

\subsection{6 -羟基黄酮及其衍生物的合成}

\subsubsection{2,5-二着基苯乙酮的合成}

取 $0.2 \mathrm{~mol}$ 对苯二酚、 $60 \mathrm{~mL}$ 冰乙酸、 $0.24 \mathrm{~mol}$ 干燥 氯化锌混合后, 油浴加热, 反应温度为 $187.0^{\circ} \mathrm{C}$. TLC 监 测反应, 颜色先变为暗红色, 回流搅拌反应 $3.0 \mathrm{~h}$ 后变为 暗红色粘稠状液体. 停止反应, 将反应液倒入冰水浴中
冷却，抽滤. 用稀盐酸反复洗涤滤饼以除去锌盐，得橙 黄色固体. 用乙酸乙酯溶解固体, 无水 $\mathrm{MgSO}_{4}$ 干燥过 夜. 回收溶剂后过柱, 用 $V$ (石油醚 $): V($ 乙酸乙酯 $)=$ $15 ： 1$ 洗脱得到 $14 \mathrm{~g}$ 浅黄色针状结晶 2,5-二羟基苯乙酮, 收率为 $76 \%$. 熔点 $206 \sim 210{ }^{\circ} \mathrm{C}$ (文献值 ${ }^{[16]}: 203 \sim 204$ $\left.{ }^{\circ} \mathrm{C}\right)$.

\subsection{2 $4 \mathrm{a} \sim 4 \mathrm{e}$ 的合成}

在反应瓶中加入 2,5 -二羟基苯乙酮 $16.2 \mathrm{mmol}$, 无 水 $\mathrm{K}_{2} \mathrm{CO}_{3} 81.0 \mathrm{mmol}$ 及干燥丙酮 $60 \mathrm{~mL} .60{ }^{\circ} \mathrm{C}$ 油浴回流 搅拌下缓慢滴加苯甲酰氯及其衍生物 $48.6 \mathrm{mmol}$, 滴毕, 反应 $24 \mathrm{~h}, \mathrm{TLC}$ 检测反应完毕. 将反应液冷却至室温, 过滤，用少量丙酮洗涤滤饼. 然后加入 $100 \mathrm{~mL} 10 \%$ 乙 酸中, 充分摚拌, 待停止冒气泡后, 过滤, 滤饼用水洗 涤至中性. 真空干燥后用丙酮重结晶得黄色针状晶体 $4 a \sim 4 e$.

1-[5-(苯甲酰氧基)-2-羟基苯基]-3-苯基-1,3-丙二酮 (4a): 淡黄色针状结晶, 收率为 $80 \%$. m.p. $204 \sim 208{ }^{\circ} \mathrm{C}$, 该化合物熔点和 ${ }^{1} \mathrm{H}$ NMR 数据没有文献报道. ${ }^{1} \mathrm{H}$ NMR $\left(400 \mathrm{MHz}, \mathrm{CDCl}_{3}\right) \delta: 15.53(\mathrm{~s}, 1 \mathrm{H}, \mathrm{C}=\mathrm{COH}), 12.05(\mathrm{~s}$, $1 \mathrm{H}, \mathrm{OH}), 8.23$ (d, $J=7.9 \mathrm{~Hz}, 2 \mathrm{H}, \mathrm{Ar}-\mathrm{H}), 7.95$ (d, $J=7.8$ $\mathrm{Hz}, 2 \mathrm{H}, \mathrm{Ar}-\mathrm{H}), 7.72 \sim 7.58(\mathrm{~m}, 2 \mathrm{H}, \mathrm{Ar}-\mathrm{H}), 7.60 \sim 7.39(\mathrm{~m}$, $5 \mathrm{H}, \mathrm{Ar}-\mathrm{H}), 7.34$ (dd, $J=9.0,2.7 \mathrm{~Hz}, 1 \mathrm{H}, \mathrm{Ar}-\mathrm{H}), 7.07$ (d, $J=9.0 \mathrm{~Hz}, 1 \mathrm{H}, \mathrm{Ar}-\mathrm{H}), 6.78(\mathrm{~s}, 1 \mathrm{H})$.

1-[5-(4'-氟苯甲酰氧基)-2-羊基-4'-氟苯基]-3-苯基1,3-丙二酮 (4b): 淡黄色针状结晶, 收率为 75\%. m.p. 206 209 ${ }^{\circ} \mathrm{C} ;{ }^{1} \mathrm{H}$ NMR $\left(400 \mathrm{MHz}, \mathrm{CDCl}_{3}\right) \delta: 15.63(\mathrm{~s}, 1 \mathrm{H}$, $\mathrm{C}=\mathrm{COH}), 12.05(\mathrm{~s}, 1 \mathrm{H}, \mathrm{OH}), 8.28(\mathrm{dd}, J=8.9,5.4 \mathrm{~Hz}$, 2H, Ar-H), 7.99 (dd, $J=8.9,5.3 \mathrm{~Hz}, 2 \mathrm{H}, \mathrm{Ar}-\mathrm{H}), 7.63$ (d, $J=2.8 \mathrm{~Hz}, 1 \mathrm{H}$, Ar-H), $7.35(\mathrm{~d}, J=2.9 \mathrm{~Hz}, 1 \mathrm{H}, \operatorname{Ar}-\mathrm{H})$, $7.27 \sim 7.19$ (m, 4H, Ar-H), 7.11 (d, $J=9.0 \mathrm{~Hz}, 1 \mathrm{H}$, Ar-H), $6.74(\mathrm{~s}, 1 \mathrm{H})$; IR (KBr) v: 3080.46, 1733.16, 1609.86, $1508.34,1449.84,1412.12,1331.62,1268.12,1229.28$, $1184.79,1157.30,1068.40,1012.40,906.19,843.36$, 806.86, $762.89 \mathrm{~cm}^{-1}$; HRMS (ESI, negative) calcd for $\mathrm{C}_{22} \mathrm{H}_{13} \mathrm{O}_{5} \mathrm{~F}_{2}\left([\mathrm{M}-\mathrm{H}]^{-}\right)$395.0737, found 395.0733.

1-[5-(4'-氯苯甲酰氧基)-2-羟基-4'-氯苯基]-3-苯基1,3-丙二酮 $(\mathbf{4 c})$ : 淡黄色针状结晶, 收率为 $75 \%$. m.p. 195.4 199 ${ }^{\circ} \mathrm{C} ;{ }^{1} \mathrm{H}$ NMR (400 MHz, $\left.\mathrm{CDCl}_{3}\right) \delta: 12.01(\mathrm{~s}$, $1 \mathrm{H}, \mathrm{OH}), 8.16(\mathrm{~d}, J=8.6 \mathrm{~Hz}, 2 \mathrm{H}, \mathrm{Ar}-\mathrm{H}), 7.88$ (d, $J=8.7$ $\mathrm{Hz}, 2 \mathrm{H}, \mathrm{Ar}-\mathrm{H}), 7.60$ (d, $J=2.8 \mathrm{~Hz}, 1 \mathrm{H}, \mathrm{Ar}-\mathrm{H}), 7.52$ (d, $J=$ $8.5 \mathrm{~Hz}, 3 \mathrm{H}, \mathrm{Ar}-\mathrm{H}), 7.46$ (d, $J=8.6 \mathrm{~Hz}, 2 \mathrm{H}, \mathrm{Ar}-\mathrm{H}), 7.08$ (d, $J=9.0 \mathrm{~Hz}, 1 \mathrm{H}, \mathrm{Ar}-\mathrm{H}), 6.73$ (s, 1H), C=COH 峰未见; IR (KBr) $v: 3419.22,1739.29,1622.67,1593.61,1566.76$, $1439.68,140130,1337.76,126619,1196.23,1092.16$, $1072.49,1014.35,881.94,797.37,748.91 \mathrm{~cm}^{-1}$; HRMS 
(ESI, negative) calcd for $\mathrm{C}_{22} \mathrm{H}_{13} \mathrm{O}_{5} \mathrm{Cl}_{2}\left([\mathrm{M}-\mathrm{H}]^{-}\right)$ 427.0146, found 427.0138.

1-[5-(4'-甲基苯甲酰氧基)-2-羟基-4'-甲基苯基]-3-苯 基-1,3-丙二酮(4d)：淡黄色针状结晶, 收率为 72\%. m.p. $181 \sim 185{ }^{\circ} \mathrm{C} ;{ }^{1} \mathrm{H}$ NMR $\left(400 \mathrm{MHz}, \mathrm{CDCl}_{3}\right) \delta: 15.62(\mathrm{~s}, 1 \mathrm{H}$, $\mathrm{C}=\mathrm{COH}), 12.10(\mathrm{~s}, 1 \mathrm{H}, \mathrm{OH}), 8.11(\mathrm{~d}, J=8.2 \mathrm{~Hz}, 2 \mathrm{H}$, Ar-H), 7.85 (d, $J=8.3 \mathrm{~Hz}, 2 \mathrm{H}, \mathrm{Ar}-\mathrm{H}), 7.61$ (d, $J=2.8 \mathrm{~Hz}$, 1H, Ar-H), 7.31 (m, 5H, Ar-H), 7.06 (d, $J=9.0 \mathrm{~Hz}, 1 \mathrm{H}$, Ar-H), 6.75 (s, $1 \mathrm{H}), 2.48$ (s, 3H, $\left.\mathrm{CH}_{3}\right), 2.43$ (s, 3H, $\mathrm{CH}_{3}$ ); IR (KBr) v: 1729.26, 1610.72, 1570.27, 1484.32, 1327.35, 1196.52, 1074.04, 802.25, $739.11 \mathrm{~cm}^{-1}$; HRMS (ESI, positive) calcd for $\mathrm{C}_{24} \mathrm{H}_{21} \mathrm{O}_{5}\left([\mathrm{M}+\mathrm{H}]^{+}\right) 389.1384$, found 389.1379 .

1-[5-(4'-甲氧基苯甲酰氧基)-2-羊基-4'-甲氧基苯 基]-3-苯基-1,3-丙二酮 (4e): 淡黄色针状结晶, 收率为 $70 \%$. m.p. $181 \sim 185{ }^{\circ} \mathrm{C} ;{ }^{1} \mathrm{H}$ NMR $\left(400 \mathrm{MHz}, \mathrm{CDCl}_{3}\right) \delta$ : $15.77(\mathrm{~s}, 1 \mathrm{H}, \mathrm{C}=\mathrm{COH}), 12.11(\mathrm{~s}, 1 \mathrm{H}, \mathrm{OH}), 8.18(\mathrm{~d}, J=8.8$ Hz, 2H, Ar-H), 7.93 (d, J=8.9 Hz, 2H, Ar-H), 7.59 (d, $J=$ $2.7 \mathrm{~Hz}, 1 \mathrm{H}, \mathrm{Ar}-\mathrm{H}), 7.08 \sim 6.94(\mathrm{~m}, 6 \mathrm{H}, \mathrm{Ar}-\mathrm{H}), 6.70(\mathrm{~s}$, $1 \mathrm{H}), 3.92\left(\mathrm{~d}, J=4.5 \mathrm{~Hz}, 3 \mathrm{H}, \mathrm{OCH}_{3}\right), 3.88\left(\mathrm{~s}, 3 \mathrm{H}, \mathrm{OCH}_{3}\right)$; IR (KBr) v: 1724.20, 1607.50, 1569.10, 1511.69, 1265.07, $1172.39,1072.85,842.86,802.93,583.56 \mathrm{~cm}^{-1}$; HRMS (ESI, positive) calcd for $\mathrm{C}_{24} \mathrm{H}_{21} \mathrm{O}_{7}\left([\mathrm{M}+\mathrm{H}]^{+}\right)$421.1282, found 421.1290 .

\subsubsection{6-羟基黄酮及其衍生物的合成}

在反应瓶中加入 $4 \mathbf{a} \sim 4 \mathrm{e} 0.1 \mathrm{mmol}$, 冰醋酸 $20 \mathrm{~mL}$ 及浓硫酸 $0.16 \mathrm{~mL}$, 控制反应温度为 $95 \sim 100{ }^{\circ} \mathrm{C}$. 搅拌 下回流反应 $1 \mathrm{~h}, \mathrm{TLC}$ 检测反应完毕, 减压回收醋酸大约 $15 \mathrm{~mL}$. 冷却至室温, 加水 $100 \mathrm{~mL}$, 有白色沉淀析出. 静置, 使沉淀析出完全, 过滤, 滤饼用水洗涤至 $\mathrm{pH}$ 值约 为 7 , 真空干燥后用无水乙醇重结晶得 6 -羟基黄醖及其 衍生物 $\mathbf{5 a} \sim 5 \mathbf{5 e}$.

6-羊基黄酮 (5a): 白色针状结晶, 收率为 75\%. m.p. 235 237 ${ }^{\circ} \mathrm{C}$ (文献值 ${ }^{[17]}: 231 \sim 233{ }^{\circ} \mathrm{C}$ ); ${ }^{1} \mathrm{H}$ NMR (400 $\left.\mathrm{MHz}, \mathrm{CD}_{3} \mathrm{OD}\right) \delta: 8.12 \sim 7.99\left(\mathrm{~m}, 2 \mathrm{H}, 2^{\prime}, 6^{\prime}-\mathrm{H}\right), 7.63 \sim 7.66$ (m, 4H, 3',4',5',7-H), 7.47 (d, $J=2.9 \mathrm{~Hz}, 1 \mathrm{H}, 8-\mathrm{H}), 7.33$ (dd, $J=9.0,2.9 \mathrm{~Hz}, 1 \mathrm{H}, 6-\mathrm{H}), 6.90$ (s, 1H, 3-H), OH 峰末 见; ESI-MS (positive) $m / z: 261.00\left([\mathrm{M}+\mathrm{Na}]^{+}\right)$.

4'-氟-6-羟基黄酮 (5b): 白色针状结晶, 收率为 75\%. m.p. $266 \sim 268{ }^{\circ} \mathrm{C}$ (文献值 ${ }^{[18]}$ : $261 \sim 262.5{ }^{\circ} \mathrm{C}$ ); ${ }^{1} \mathrm{H}$ NMR (400 MHz, CD $\left.\mathrm{CD}_{3} \mathrm{OD}\right) \delta: 8.03(\mathrm{dd}, J=9.1,5.2 \mathrm{~Hz}, 2 \mathrm{H}$, 2',6'-H), 7.54 (d, $J=9.0 \mathrm{~Hz}, 1 \mathrm{H}, 5-\mathrm{H}), 7.35$ (d, $J=3.0 \mathrm{~Hz}$, 1H, 7-H), $7.29 \sim 7.18$ (m, 3H, 3', $\left.4^{\prime}, 8-\mathrm{H}\right), 6.77$ (s, 1H, 3-H), $\mathrm{OH}$ 峰未见; ESI-MS (positive) $\mathrm{m} / z: 279.00\left([\mathrm{M}+\mathrm{Na}]^{+}\right)$.

4'-氯-6-着基黄酮 $(\mathbf{5 c})$ : 白色针状结晶, 收率为 75\%. m.p. $283 \sim 286{ }^{\circ} \mathrm{C}$, 该化合物熔点和 ${ }^{1} \mathrm{H}$ NMR 没有文献 报道. ${ }^{1} \mathrm{H}$ NMR $\left(400 \mathrm{MHz}, \mathrm{CD}_{3} \mathrm{OD}\right) \delta: 8.09(\mathrm{~d}, J=8.0 \mathrm{~Hz}$, $\left.2 \mathrm{H}, 2^{\prime}, 6^{\prime}-\mathrm{H}\right), 7.65$ (dd, $\left.J=15.3,8.5 \mathrm{~Hz}, 3 \mathrm{H}, 3^{\prime}, 4^{\prime}, 5^{\prime}-\mathrm{H}\right)$, $7.48(\mathrm{~d}, J=3.0 \mathrm{~Hz}, 1 \mathrm{H}, 7-\mathrm{H}), 7.39 \sim 7.29(\mathrm{~m}, 1 \mathrm{H}, 8-\mathrm{H})$, 6.92 (d, $J=1.0 \mathrm{~Hz}, 1 \mathrm{H}, 3-\mathrm{H})$; OH 峰未见; ESI-MS (positive) $m / z: 295.00\left([\mathrm{M}+\mathrm{Na}]^{+}\right)$.

4'-甲基-6-羊基黄酮 (5d): 淡黄色结晶, 收率为 70\%. m.p. $282 \sim 285{ }^{\circ} \mathrm{C}$, 该化合物熔点和 ${ }^{1} \mathrm{H}$ NMR 没有文献 报道. ${ }^{1} \mathrm{H}$ NMR (400 MHz, $\left.\mathrm{CD}_{3} \mathrm{OD}\right) \delta: 7.97(\mathrm{~d}, J=8.2 \mathrm{~Hz}$, 2H, 2', $\left.6^{\prime}-\mathrm{H}\right), 7.65$ (d, $\left.J=9.0 \mathrm{~Hz}, 1 \mathrm{H}, 7-\mathrm{H}\right), 7.44$ (dd, $J=$ $\left.16.5,5.5 \mathrm{~Hz}, 3 \mathrm{H}, 3^{\prime}, 4^{\prime}, 5^{\prime}-\mathrm{H}\right), 7.32$ (dd, $J=9.0,3.0 \mathrm{~Hz}, 1 \mathrm{H}$, 8-H), 6.87 (s, 1H, 3-H), OH 峰未见; ESI-MS (positive) $m / z: 275.00\left([\mathrm{M}+\mathrm{Na}]^{+}\right)$.

4'-甲氧基-6-羟基黄酮 (5e): 淡黄色结晶, 收率为 $70 \%$. m.p. 254 257 ${ }^{\circ} \mathrm{C}$ (文献值 ${ }^{[19]}: 249{ }^{\circ} \mathrm{C}$ ); ${ }^{1} \mathrm{H}$ NMR $\left(400 \mathrm{MHz},\left(\mathrm{CD}_{3}\right)_{2} \mathrm{CO}\right) \delta: 8.00\left(\mathrm{~d}, J=8.9 \mathrm{~Hz}, 2 \mathrm{H}, 2^{\prime}, 6^{\prime}-\mathrm{H}\right)$, $7.60(\mathrm{~d}, J=9.0 \mathrm{~Hz}, 1 \mathrm{H}, 7-\mathrm{H}), 7.42(\mathrm{~d}, J=3.0 \mathrm{~Hz}, 1 \mathrm{H}$, 8-H), 7.27 (dd, $\left.J=9.0,3.0 \mathrm{~Hz}, 1 \mathrm{H}, 5^{\prime}-\mathrm{H}\right), 7.10$ (d, $J=9.0$ $\left.\mathrm{Hz}, 2 \mathrm{H}, 3^{\prime}, 4^{\prime}-\mathrm{H}\right), 6.78$ (s, 1H, 3-H), 3.89 (s, 3H, $\left.\mathrm{OCH}_{3}\right)$, $\mathrm{OH}$ 峰末见; ESI-MS (positive) $\mathrm{m} / z: 291.00$ ([M+Na $]^{+}$).

\subsubsection{6-羟基黄酮衍生物 $6 \mathrm{~b}$ 和 $6 \mathrm{~d}$ 的合成}

在圆底烧瓶中加入 $\mathbf{5 b}$ 或 $\mathbf{5 d} 0.1 \mathrm{mmol}$, 无水 $\mathrm{K}_{2} \mathrm{CO}_{3}$ $0.3 \mathrm{mmol}$ 及丙酮 $20 \mathrm{~mL}, 60{ }^{\circ} \mathrm{C}$ 油浴回流搅拌下缓慢滴加 苯甲酰氯 $0.3 \mathrm{mmol}$, 滴毕, 回流反应 $30 \mathrm{~min}$. TLC 跟踪 检测, 原料反应完全后, 减压回收溶剂. 于固体中加入 稀盐酸使 $\mathrm{pH}$ 值约为 3 , 过滤. 滤饼用水洗至中性, 真空 干燥后用无水乙醇重结晶得白色结晶 $\mathbf{6 b}$ 和 $\mathbf{6 d}$.

6-苯甲酰氧基-4'-氟黄酮 (6b): 白色结晶, 收率为 70\%. m.p. 238 $240{ }^{\circ} \mathrm{C} ;{ }^{1} \mathrm{H}$ NMR (400 MHz, $\left.\mathrm{CDCl}_{3}\right) \delta$ : $8.21\left(\mathrm{t}, J=15.2 \mathrm{~Hz}, 2 \mathrm{H}, 2^{\prime}, 6^{\prime}-\mathrm{H}\right), 8.06$ (s, 1H, 5-H), 7.96 (dd, $J=7.4,5.3 \mathrm{~Hz}, 2 \mathrm{H}, \mathrm{Ar}-\mathrm{H}), 7.69(\mathrm{~d}, J=9.2 \mathrm{~Hz}, 2 \mathrm{H}$, Ar-H), $7.64 \sim 7.46$ (m, 3H, 7,8-H, Ar-H), 7.24 (d, $J=8.3$ $\left.\mathrm{Hz}, 2 \mathrm{H}, 3^{\prime}, 4^{\prime}-\mathrm{H}\right), 6.79$ (s, 1H, 3-H); IR (KBr) v: 3077.97, 1739.22, 1636.04, 1511.83, 1460.00, 1262.59, 1234.13, 1129.39, 1086.14, 911.53, 836.50, 703.95, 591.24, 554.62, $500.57 \mathrm{~cm}^{-1}$; HRMS (ESI, positive) calcd for $\mathrm{C}_{22} \mathrm{H}_{14} \mathrm{O}_{4} \mathrm{~F}$ $\left([\mathrm{M}+\mathrm{H}]^{+}\right)$361.0871, found 361.0867.

6-苯甲酰氧基-4'-甲基黄酮(6d): 白色结晶, 收率为 $65 \%$. m.p. $218 \sim 224{ }^{\circ} \mathrm{C} ;{ }^{1} \mathrm{H}$ NMR $\left(400 \mathrm{MHz}, \mathrm{CDCl}_{3}\right) \delta$ : $8.23\left(\mathrm{~d}, J=7.2 \mathrm{~Hz}, 2 \mathrm{H}, 2^{\prime}, 6^{\prime}-\mathrm{H}\right), 8.06(\mathrm{~d}, J=2.7 \mathrm{~Hz}, 1 \mathrm{H}$, $5-\mathrm{H}), 7.85(\mathrm{~d}, J=8.2 \mathrm{~Hz}, 2 \mathrm{H}, \operatorname{Ar}-\mathrm{H}), 7.67(\mathrm{~d}, J=9.1 \mathrm{~Hz}$, $2 \mathrm{H}, \mathrm{Ar}-\mathrm{H}), 7.63 \sim 7.51$ (m, 3H, 7,8-H, Ar-H), 7.35 (d, $J=$ $\left.8.1 \mathrm{~Hz}, 2 \mathrm{H}, 3^{\prime}, 4^{\prime}-\mathrm{H}\right), 6.83$ (s, 1H, 3-H), 2.46 (s, 3H, $\left.\mathrm{CH}_{3}\right)$; IR (KBr) v: 1736.19, 1636.5 9, 1443.39, 1362.58, 1263.49, 1174.30, 1086.34, 1057.85, 903.68, 857.79, 818.13, 
703.62, 590.32, 555.33, $481.88 \mathrm{~cm}^{-1}$; ESI-MS (positive) $m / z: 379.08\left([\mathrm{M}+\mathrm{Na}]^{+}\right), 735.83([2 \mathrm{M}+\mathrm{Na}])^{+} ; \mathrm{HRMS}$ (ESI, positive) calcd for $\mathrm{C}_{23} \mathrm{H}_{17} \mathrm{O}_{4}\left([\mathrm{M}+\mathrm{H}]^{+}\right) 357.3849$, found 357.3845 .

\subsection{7 -羟基黄酮及其衍生物的合成}

\subsection{1 化合物 $\mathbf{1 0 a} \sim 10 \mathrm{e}$ 的合成}

\section{合成方法参考第 3.2 节.}

7-羟基黄酮(10a): 白色针状结晶, 收率为 $85 \%$. m.p. 244 246 ${ }^{\circ} \mathrm{C}$ (文献值 ${ }^{[20]:}: 240 \sim 241{ }^{\circ} \mathrm{C}$ ); ${ }^{1} \mathrm{H}$ NMR (400 $\left.\mathrm{MHz}, \mathrm{CD}_{3} \mathrm{OD}\right) \delta: 8.08 \sim 8.00\left(\mathrm{~m}, 3 \mathrm{H}, 2^{\prime}, 6^{\prime}, 5-\mathrm{H}\right), 7.64 \sim$ 7.54 (m, 3H, 3',4',5'-H), 7.03 (d, $J=2.2 \mathrm{~Hz}, 1 \mathrm{H}, 8-\mathrm{H}), 6.98$ (dd, $J=8.8,2.2 \mathrm{~Hz}, 1 \mathrm{H}, 6-\mathrm{H}), 6.84$ (s, 1H, 3-H), OH 峰未 见; ESI-MS (positive) $m / z: 261.00\left([\mathrm{M}+\mathrm{Na}]^{+}\right)$.

4'-氟-7-羟基黄酮 (10b): 黄色针状结晶, 收率为 $85 \%$. m.p. $250 \sim 254{ }^{\circ} \mathrm{C}$ (文献值 ${ }^{[21]}: 246 \sim 248{ }^{\circ} \mathrm{C}$ ); ${ }^{1} \mathrm{H}$ NMR (400 MHz, Acetone- $\left.d_{6}\right) \delta: 9.74(\mathrm{~s}, 1 \mathrm{H}, \mathrm{OH}), 8.29 \sim$ $8.12\left(\mathrm{~m}, 2 \mathrm{H}, 2^{\prime}, 66^{\prime}-\mathrm{H}\right), 8.03(\mathrm{~d}, J=8.7 \mathrm{~Hz}, 1 \mathrm{H}, 5-\mathrm{H}), 7.42(\mathrm{t}$, $\left.J=8.8 \mathrm{~Hz}, 2 \mathrm{H}, 3^{\prime}, 4^{\prime}-\mathrm{H}\right), 7.12(\mathrm{~d}, J=2.2 \mathrm{~Hz}, 1 \mathrm{H}, 8-\mathrm{H}), 7.05$ (dd, $J=8.7,2.3 \mathrm{~Hz}, 1 \mathrm{H}, 6-\mathrm{H}), 6.79$ (s, 1H, 3-H); ESI-MS (positive) $m / z: 257.00\left([\mathrm{M}+\mathrm{Na}]^{+}\right.$).

4'-氯-7-羊基黄酮 (10c): 白色针状结晶, 收率为 $85 \%$. m.p. $284.9 \sim 286{ }^{\circ} \mathrm{C}$ (文献值 ${ }^{[22]}: 280 \sim 282{ }^{\circ} \mathrm{C}$ ); ${ }^{1} \mathrm{H}$ NMR (400 MHz, Acetone- $\left.d_{6}\right) \delta: 9.80(\mathrm{~s}, 1 \mathrm{H}, \mathrm{OH}), 8.14$ (d, $\left.J=8.7 \mathrm{~Hz}, 2 \mathrm{H}, 2^{\prime}, 6^{\prime}-\mathrm{H}\right), 8.03(\mathrm{~d}, J=8.7 \mathrm{~Hz}, 1 \mathrm{H}, 5-\mathrm{H}), 7.68$ (d, $\left.J=8.6 \mathrm{~Hz}, 2 \mathrm{H}, 3^{\prime}, 4^{\prime}-\mathrm{H}\right), 7.12(\mathrm{~d}, J=2.2 \mathrm{~Hz}, 1 \mathrm{H}, 8-\mathrm{H})$, 7.05 (dd, $J=8.7,2.2 \mathrm{~Hz}, 1 \mathrm{H}, 6-\mathrm{H}), 6.83$ (s, 1H, 3-H).

4'-甲基-7-羊基黄酮(10d): 淡黄色针状结晶, 收率 为 $85 \%$. m.p. $282 \sim 284{ }^{\circ} \mathrm{C}$ (文献值 ${ }^{[17]}$ : $278 \sim 280{ }^{\circ} \mathrm{C}$ ); ${ }^{1} \mathrm{H}$ NMR (400 MHz, Acetone- $\left.d_{6}\right) \delta: 9.67(\mathrm{~s}, 1 \mathrm{H}, \mathrm{OH}), 8.01$ (dd, $\left.J=9.9,8.6 \mathrm{~Hz}, 3 \mathrm{H}, 2^{\prime}, 6^{\prime}, 5-\mathrm{H}\right), 7.45$ (d, $J=8.1 \mathrm{~Hz}, 2 \mathrm{H}$, $\left.3^{\prime}, 4^{\prime}-\mathrm{H}\right), 7.11(\mathrm{~d}, J=2.2 \mathrm{~Hz}, 1 \mathrm{H}, 8-\mathrm{H}), 7.04$ (dd, $J=8.7$, $2.3 \mathrm{~Hz}, 1 \mathrm{H}, 6-\mathrm{H}), 6.76(\mathrm{~s}, 1 \mathrm{H}, 3-\mathrm{H}), 2.48\left(\mathrm{~s}, 3 \mathrm{H}, \mathrm{CH}_{3}\right)$. ESI-MS (positive) $m / z: 275.00\left([\mathrm{M}+\mathrm{H}]^{+}\right)$.

4'-甲氧基-7-羊基黄酮(10e): 淡黄色针状结晶, 收 率为 $85 \%$. m.p. $265 \sim 267{ }^{\circ} \mathrm{C}$ (文献值 ${ }^{[23]}$ : $263 \sim 264{ }^{\circ} \mathrm{C}$ ); ${ }^{1} \mathrm{H}$ NMR (400 MHz, Acetone- $\left.d_{6}\right) \delta: 8.07 \sim 8.03(\mathrm{~m}, 2 \mathrm{H}$, $\left.2^{\prime}, 6^{\prime}-\mathrm{H}\right), 8.00(\mathrm{~d}, J=8.7 \mathrm{~Hz}, 1 \mathrm{H}, 5-\mathrm{H}), 7.18 \sim 7.12(\mathrm{~m}, 2 \mathrm{H}$, $\left.3^{\prime}, 4^{\prime}-\mathrm{H}\right), 7.08(\mathrm{~d}, J=2.2 \mathrm{~Hz}, 1 \mathrm{H}, 8-\mathrm{H}), 7.01$ (dd, $J=8.7$, $2.3 \mathrm{~Hz}, 1 \mathrm{H}, 6-\mathrm{H}), 6.69$ (s, 1H, 3-H), 3.94 (s, 3H, $\mathrm{OCH}_{3}$ ), $\mathrm{OH}$ 峰未见; ESI-MS (positive) $\mathrm{m} / z: 291.00\left([\mathrm{M}+\mathrm{Na}]^{+}\right.$).

\subsubsection{1b 和 $11 \mathrm{e}$ 的合成}

合成方法参考第 3.2 .4 节.

7-苯甲酰氧基-4'-氟黄酮(11b): 白色结晶, 收率为 $74 \%$. m.p. $238 \sim 240{ }^{\circ} \mathrm{C}$; 此化合物未见文献报道. ${ }^{1} \mathrm{H}$ NMR (400 MHz, $\left.\mathrm{CDCl}_{3}\right) \delta: 8.31(\mathrm{~d}, J=8.7 \mathrm{~Hz}, 1 \mathrm{H}, 5-\mathrm{H})$,
8.24 (d, $\left.J=8.0 \mathrm{~Hz}, 2 \mathrm{H}, 2^{\prime}, 6^{\prime}-\mathrm{H}\right), 7.93$ (dd, $J=7.5,5.2 \mathrm{~Hz}$, $\left.2 \mathrm{H}, 3^{\prime}, 4^{\prime}-\mathrm{H}\right), 7.70$ (t, $\left.J=6.9 \mathrm{~Hz}, 1 \mathrm{H}, 8-\mathrm{H}\right), 7.56(\mathrm{t}, J=7.2$ $\mathrm{Hz}, 3 \mathrm{H}, \mathrm{Ar}-\mathrm{H}), 7.32$ (d, $J=8.7 \mathrm{~Hz}, 1 \mathrm{H}, 6-\mathrm{H}), 7.22$ (d, $J=$ $8.3 \mathrm{~Hz}, 2 \mathrm{H}, \mathrm{Ar}-\mathrm{H}), 6.80$ (s, 1H, 3-H); IR (KBr) v: 1741.89, 1660. 99, 1601.32, 1506.20, 1439.03, 1416.04, 1368.40, $1267.17,1234.18,1150.77,1090.79,1056.45,1024.01$, 836.46, 701.16, $675.54 \mathrm{~cm}^{-1}$; ESI-MS (positive) $\mathrm{m} / \mathrm{z}$ : $383.00(\mathrm{M}+\mathrm{Na})^{+}$; HRMS (ESI, positive) calcd for $\mathrm{C}_{22} \mathrm{H}_{14} \mathrm{O}_{4} \mathrm{~F}\left([\mathrm{M}+\mathrm{H}]^{+}\right)$361.3486; found 361.3476

7-苯甲酰氧基-4'-甲氧基黄酮(11e): 白色针状结晶, 收率为 78\%. m.p. $200 \sim 204{ }^{\circ} \mathrm{C} ;{ }^{1} \mathrm{H}$ NMR $(400 \mathrm{MHz}$, $\left.\mathrm{CDCl}_{3}\right) \delta: 8.34 \sim 8.27(\mathrm{~d}, J=9.3 \mathrm{~Hz}, 1 \mathrm{H}, 5-\mathrm{H}), 8.24(\mathrm{~d}$, $\left.J=7.3 \mathrm{~Hz}, 2 \mathrm{H}, 2^{\prime}, 6^{\prime}-\mathrm{H}\right), 7.89\left(\mathrm{~d}, J=8.8 \mathrm{~Hz}, 2 \mathrm{H}, 3^{\prime}, 4^{\prime}-\mathrm{H}\right)$, $7.72 \sim 7.66(\mathrm{~m}, 1 \mathrm{H}, 8-\mathrm{H}), 7.60 \sim 7.51(\mathrm{~m}, 4 \mathrm{H}, \mathrm{Ar}-\mathrm{H}), 7.04$ (d, $J=8.9 \mathrm{~Hz}, 2 \mathrm{H}, \mathrm{Ar}-\mathrm{H}), 6.79$ (s, 1H, 3-H), 3.90 (s, 3H, $\left.\mathrm{OCH}_{3}\right)$; IR $v: 1747.17,1654.52,1604.89,1513.36$, $1440.19,1370.19,1241.32,1185.00,1151.57,1051.80$, $1023.59,877.58,829.47,701.09,507.90 \mathrm{~cm}^{-1}$. ESI-MS (positive) $\mathrm{m} / z: 395.00\left([\mathrm{M}+\mathrm{Na}]^{+}\right)$; HRMS (ESI, positive) calcd for $\mathrm{C}_{23} \mathrm{H}_{17} \mathrm{O}_{5}\left([\mathrm{M}+\mathrm{H}]^{+}\right)$373.3843, found 373.3835 .

\subsection{3 化合物 12 的合成}

在圆底烧瓶中加入 7-美基黄酮 $0.103 \mathrm{~g}(0.43 \mathrm{mmol})$, 无水 $\mathrm{K}_{2} \mathrm{CO}_{3} 0.108 \mathrm{~g}(1.3 \mathrm{mmol})$ 及丙酮 $20 \mathrm{~mL}$, 再加 1-氯3 -溴丙烷 $0.074 \mathrm{~g}(0.47 \mathrm{mmol}) .60{ }^{\circ} \mathrm{C}$ 油浴回流反应 $12 \mathrm{~h}$, TLC 跟踪检测, 原料反应完全后, 过滤. 滤饼用少量丙 酮淋洗, 合并滤液, 减压回收溶剂后, 用无水乙醇重结 晶得白色结晶 7-(3-氯丙氧基)-黄酮 $0.11 \mathrm{~g}$, 收率 81\%. 熔点 $121 \sim 124{ }^{\circ} \mathrm{C}$.

\subsection{4 化合物 13 的合成}

称取诺氟沙星 $0.1 \mathrm{mmol} 、 7$-(3-氯丙氧基)-黄酮 0.08 $\mathrm{mmol} 、 0.5 \mathrm{mmol} \mathrm{K}_{2} \mathrm{CO}_{3}$ 和少量 $\mathrm{KCl}$ 加入反应瓶中. 20 $\mathrm{mL}$ 丙酮作溶剂, $60{ }^{\circ} \mathrm{C}$ 油浴回流反应 $36 \mathrm{~h}$. TLC 跟踪检 测, 有新的天蓝色荧光斑点生成, 极性介于 7-(3-氯丙氧 基)-黄酮和诺氟沙星之间. 反应停止，回收溶剂，用稀 盐酸酸化使溶液 $\mathrm{pH}$ 值约为 3 , 过滤. 滤饼用水洗至中 性，真空干燥后用丙酮重结晶得到灰白色固体 13. 灰白 色无定形粉末，收率为 76\%. ${ }^{1} \mathrm{H}$ NMR $(400 \mathrm{MHz}$, Acetone- $\left.d_{6}\right) \delta: 15.07(\mathrm{~s}, 1 \mathrm{H}, \mathrm{COOH}), 9.44(\mathrm{~s}, 1 \mathrm{H}$, Norfloxacin 2-H), 8.45 (d, $J=12.4 \mathrm{~Hz}, 1 \mathrm{H}$, Norfloxacin 5-H), $\delta: 8.14 \sim$ $8.00\left(\mathrm{~m}, 3 \mathrm{H}\right.$, flavone $\left.2^{\prime}, 6^{\prime}, 5-\mathrm{H}\right), 7.74 \sim 7.56(\mathrm{~m}, 3 \mathrm{H}$, flavone $\left.3^{\prime}, 4^{\prime}, 5^{\prime}-\mathrm{H}\right), 7.48(\mathrm{~d}, J=7.4 \mathrm{~Hz}, 1 \mathrm{H}$, Norfloxacin $8-\mathrm{H})$, $7.13(\mathrm{~d}, J=2.2 \mathrm{~Hz}, 1 \mathrm{H}$, flavone 8-H), 7.00 (dd, $J=8.8,2.2$ $\mathrm{Hz}, 1 \mathrm{H}$, flavone 6-H), 6.94 (s, 1H, flavone 3-H), 4.88 (q, $J=7.3 \mathrm{~Hz}, 2 \mathrm{H}$, Norfloxacin 1- $\left.\mathrm{CH}_{2}\right), 4.45$ (t, $J=4.72 \mathrm{~Hz}$, $\left.2 \mathrm{H}, \mathrm{OCH}_{2}\right), 4.34 \sim 3.94\left(\mathrm{~m}, 8 \mathrm{H}\right.$, Norfloxacin $\left.2^{\prime}, 3^{\prime}, 5^{\prime}, 6^{\prime}-\mathrm{H}\right)$, 
$3.88\left(\mathrm{t}, J=4.60 \mathrm{~Hz}, 2 \mathrm{H}, \mathrm{NCH}_{2}\right), 2.41 \sim 2.36(\mathrm{~m}, 2 \mathrm{H}$, $\mathrm{NCH}_{2} \mathrm{CH}_{2} \mathrm{CH}_{2} \mathrm{O}$ ), 1.90 (t, $J=7.3 \mathrm{~Hz}, 3 \mathrm{H}$, Norfloxacin 1- $\mathrm{CH}_{3}$ ). HRMS (ESI, positive) calcd for $\mathrm{C}_{34} \mathrm{H}_{33} \mathrm{O}_{6} \mathrm{~N}_{3} \mathrm{~F}$ $\left([\mathrm{M}+\mathrm{H}]^{+}\right)$598.2348, found 598.2338.

\subsection{MTT 法体外抗肿瘤活性实验}

按照文献[9]方法, 取对数生长期的 Hela 细胞, 制成 细胞密度为 $5 \times 10^{4}$ 个 $/ \mathrm{mL}$, 接种于 96 孔培养板中. 每孔 RPMI-1640 培养基 $200 \mu \mathrm{L}$, 置于 $37{ }^{\circ} \mathrm{C}, 5 \%$ 的 $\mathrm{CO}_{2}$ 及 $90 \%$ 湿度条件下无血清孵育 $48 \mathrm{~h}$. 细胞完全贴壁后, 更换新 鲜培养液. 设空白对照组(普通培养基)、实验组(10, 20, $50,100 \mu \mathrm{g} / \mathrm{mL}$ 药物浓度梯度), 每个处理组设 3 个平行 孔, 加在 96 孔板内. 每孔加 $20 \mu \mathrm{L}$, 加药完毕后放回睬 箱继续培养 $48 \mathrm{~h}$ 后, 吸弃上清液, 每孔加入新鲜配制的 MTT 溶液 $(5 \mathrm{mg} / \mathrm{mL}) 20 \mu \mathrm{L}$, 在 $37{ }^{\circ} \mathrm{C}$ 恒温培养箱中继续 培养 4 h. 终止培养, 弃除孔内上清液, 每孔加入 DMSO $200 \mu \mathrm{L}$, 平板摇床振荡 $30 \mathrm{~min}(60$ 次 $/ \mathrm{min}$ ) 使甲㬺沉淀充 分溶解. 用酶联免疫检测仪在 $490 \mathrm{~nm}$ 波长处测定各孔 的吸光度 (A 值), 重复测试 3 次, 取平均值为最终结果. 经培养的癌细胞通过孔中药物抑制后, 按下列公式计算 细胞抑制率.

细胞抑制率 $=($ 对照组 $\mathrm{A}$ 值一实验组 $\mathrm{A}$ 值) $/$ 对照组 $\mathrm{A}$ 值 $\times 100 \%$

通过回归计算, 求出半数抑制浓度, 即细胞增殖抑 制率为 $50 \%$ 时的药物浓度 $\left(\mathrm{IC}_{50}\right.$ 值).

\section{References}

[1] Rafi, M. M.; Rosen, R. T.; Vassil, A. Anticancer Res. 2000, 20, 2653.

[2] Silvia, G.; Andrea, C.; Angela, R.; Federica, B.; Lorna, P.; Anja, P.; Rolf, W. H.; Maurizio, R.; Alessandra, B. J. Med. Chem. 2006, 49, 4777.
[3] Xia, L.; Chi, B. C.; Sung, W. J.; Sompol, P.; Huang, J. J.; Kunyan, H.; Lien, H. P.; Stefan F.; Ge, X.; Jia, Y. H.; Hongbo, R. L.; Ye, K. Q.J.Med.Chem. 2010, 53, 8274.

[4] Yan, X.; Liu, H. Q.; Zou, Y. Q.; Ren, Z. H. Chin. J. Org. Chem. 2008, 28, 1534 (in Chinese). (延尔, 刘会青, 邹永青, 任占华, 有机化学, 2008, 28, 1534.)

[5] Wang, X. J.; Liu, J. L. Chem. Res. Appl. 2013, 25, 1491 (in Chinese). (王学军, 刘建利, 化学研究与应用, 2013, 25, 1491.)

[6] Bakerr, W. J. Chem. Soc. 1933, 55, 1381.

[7] Lee, J. I.; Son, H. S.; Park, H. Bull. Korean Chem. Soc. 2004, 25(12), 1945.

[8] Chee, C. F.; Buckle, M. C.; Rahman, N. A. Tetrahedron Lett. 2011, 52,3120 .

[9] Rafi, M. M.; Rosen, R, T.; Vassil, A. Anticancer Res. 2000, 20, 2653.

[10] Zhang, Q. Z.; Yang, X. S.; Hao, X. J. Chin. Tradit. Herb. Drugs 2008, 39, 304 (in Chinese).

(张前军, 杨小生, 郝小江, 中草药, 2008, 39, 304.)

[11] Lechner, D.; Gibbons, S.; Bucar, F. Phytochem. Lett. 2008, 1, 71.

[12] Lee, Y. S.; Kang, O. H.; Choi, J. G. J. Microbiol. 2008, 46, 283.

[13] Chang, P. C.; Li, H. Y.; Tang, H. J. J. Microbiol. Immunol. Infect. 2007, 40, 56 .

[14] Stapleton, P. D.; Shah, S.; Anderson, J. C. Int. J. Antimicrob. Agents 2004, 23, 462.

[15] Nong, C. Z.; Ye, H. H.; Wang, L. Chin. J. Hosp. Pharm. 2011, 31, 750 (in Chinese). (农朝赞, 叶海洪, 王丽, 中国医院药学杂志, 2011, 31, 750.)

[16] Chen, A. H.; Kuo, W. B.; Chen, C. W. J. Chin. Chem. Soc. 2004, $51,1389$.

[17] Tang, L. J.; Zhang, S. F.; Yang, J. Z.; Gao, W. T.; Cui, J. Org. Prep. Proc. Int. 2004, 36, 453.

[18] Jiraporn, U.; Chanpen, W.; Weerasak, S.; Patcharawee, N. J. Mol. Struct. 2011, 1001, 152.

[19] Tara, C. C.; Krishnasami, V. J. Chem. Soc. 1933, 1073.

[20] Bhosale, R. S.; Sarda, S. R.; Giram, R. P. J. Iran. Chem. Soc. 2009, $6,519$.

[21] Liu, X. P.; Wang, Y.; Lan, H. Y. Chem. Res. Chin. Univ. 2010, 26, 268.

[22] Wu, Z.; Jiang, L.; Chen, H. J. Chem. Res. 2009, 3, 195.

[23] Wilson, B. J. Chem. Soc. 1933, 1381. 